Acta Crystallographica Section E

Structure Reports

Online

ISSN 1600-5368

\section{2,5-Dihydroxyterephthalic acid dihydrate}

\section{Po-Wen Cheng, Chi-Feng Cheng, Yeh Chun-Ting and Chia-Her Lin*}

Department of Chemistry, Chung-Yuan Christian University, Chung-Li 320, Taiwan Correspondence e-mail: chiaher@cycu.edu.tw

Received 25 June 2010; accepted 30 June 2010

Key indicators: single-crystal X-ray study; $T=295 \mathrm{~K}$; mean $\sigma(\mathrm{C}-\mathrm{C})=0.004 \AA$; $R$ factor $=0.061 ; w R$ factor $=0.193 ;$ data-to-parameter ratio $=16.5$.

The title compound, $\mathrm{C}_{8} \mathrm{H}_{6} \mathrm{O}_{6} \cdot 2 \mathrm{H}_{2} \mathrm{O}$, was obtained by accident within a project on the synthesis of metal-organic coordination polymers by the reaction of $\mathrm{LiOH}$ with 2,5-dihydroxyterephthalic acid under solvothermal conditions. The asymmetric unit consists of half a 2,5-dihydroxyterephthalic acid molecule located on a centre of inversion and one solvent water molecule that occupies a general position. The 2,5dihydroxyterephthalic acid molecules are connected to the water molecules via $\mathrm{O}-\mathrm{H} \cdots \mathrm{O}$ hydrogen bonding to form a layer in the $a b$ plane.

\section{Related literature}

For genernal background to supramolecular assembly and crystal engineering, see: Kitagawa et al. (2004).

\section{Experimental}

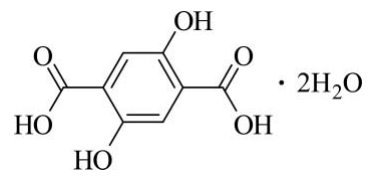

Crystal data

$\mathrm{C}_{8} \mathrm{H}_{6} \mathrm{O}_{6} \cdot 2 \mathrm{H}_{2} \mathrm{O}$

$M_{r}=234.16$

Monoclinic, $P 2_{1} / c$

$$
\begin{aligned}
& \beta=103.03(1)^{\circ} \\
& V=487.68(17) \AA^{3} \\
& Z=2 \\
& \text { Mo } K \alpha \text { radiation }
\end{aligned}
$$

Data collection

Bruker APEXII CCD diffractometer

Absorption correction: multi-scan (SADABS; Bruker, 2009)

$T_{\min }=0.945, T_{\max }=0.963$

\section{Refinement}

$R\left[F^{2}>2 \sigma\left(F^{2}\right)\right]=0.061$

$w R\left(F^{2}\right)=0.193$

$S=1.02$

1208 reflections $\mu=0.15 \mathrm{~mm}^{-1}$

$T=295 \mathrm{~K}$

$0.25 \times 0.20 \times 0.20 \mathrm{~mm}$

4475 measured reflections 1208 independent reflections 589 reflections with $I>2 \sigma(I)$ $R_{\text {int }}=0.080$

73 parameters

$\mathrm{H}$-atom parameters constrained

$\Delta \rho_{\max }=0.36{\mathrm{e} \AA^{-3}}^{-3}$

$\Delta \rho_{\min }=-0.32{\mathrm{e} \AA^{-3}}^{-3}$

Table 1

Hydrogen-bond geometry $\left(\AA{ }^{\circ}\right)$.

\begin{tabular}{lllll}
\hline$D-\mathrm{H} \cdots A$ & $D-\mathrm{H}$ & $\mathrm{H} \cdots A$ & $D \cdots A$ & $D-\mathrm{H} \cdots A$ \\
\hline $\mathrm{O} 1-\mathrm{H} 1 A \cdots \mathrm{O} 3^{\mathrm{i}}$ & 0.82 & 1.88 & $2.597(3)$ & 146 \\
$\mathrm{O} 2-\mathrm{H} 2 B \cdots \mathrm{O} 1 W^{\mathrm{ii}}$ & 0.82 & 1.74 & $2.561(3)$ & 177 \\
$\mathrm{O} 1 W-\mathrm{H} 1 W B \cdots \mathrm{O} 1^{\mathrm{iii}}$ & 0.85 & 1.94 & $2.786(3)$ & 175.0 \\
$\mathrm{O} 1 W-\mathrm{H} 1 W A \cdots{ }^{\text {iv }}$ & 0.85 & 2.04 & $2.809(3)$ & 150.4 \\
\hline
\end{tabular}

Symmetry codes: (i) $-x,-y+1,-z+2$; (ii) $x, y, z-1$; (iii) $-x+1,-y+1,-z+2$; (iv) $x,-y+\frac{3}{2}, z+\frac{1}{2}$.

Data collection: APEX2 (Bruker, 2009); cell refinement: SAINT (Bruker, 2009); data reduction: $S A I N T$; $\operatorname{program}(\mathrm{s})$ used to solve structure: SHELXS97 (Sheldrick, 2008); program(s) used to refine structure: SHELXL97 (Sheldrick, 2008); molecular graphics: SHELXTL (Sheldrick, 2008); software used to prepare material for publication: SHELXTL.

The authors gratefully acknowledge financial support from the National Science Council, Taiwan, and also funding as a CYCU Distinctive Research Area project (grant No. CYCU98-CR-CH).

Supplementary data and figures for this paper are available from the IUCr electronic archives (Reference: NC2191).

\title{
References
}

Bruker (2009). APEX2, SADABS and SAINT. Bruker AXS Inc., Madison, Wisconsin, USA.

Kitagawa, S., Kitaura, R. \& Noro, S. (2004). Angew. Chem. Int. Ed. 43, 2334 2338.

Sheldrick, G. M. (2008). Acta Cryst. A64, 112-122. 


\section{supporting information}

Acta Cryst. (2010). E66, o1928 [https://doi.org/10.1107/S1600536810025766]

\section{2,5-Dihydroxyterephthalic acid dihydrate}

\section{Po-Wen Cheng, Chi-Feng Cheng, Yeh Chun-Ting and Chia-Her Lin}

\section{S1. Experimental}

The solvothermal reactions were carried out in Teflon-lined digestion bombs (internal volume of $23 \mathrm{ml}$ ) under autogenously pressure by heating the reaction mixtures followed by slow cooling at $6 \mathrm{~K} \mathrm{~h}^{-1}$ to room temperature. Crystals of the title compound were obtained from the reaction of 2,5-dihydroxyterephthalic acid $\left(\mathrm{C}_{8} \mathrm{H}_{4} \mathrm{O}_{6}, 0.198 \mathrm{~g}, 1.0 \mathrm{mmol}\right)$ with $\mathrm{Li}(\mathrm{OH})(0.048 \mathrm{~g}, 2.0 \mathrm{mmol})$ in $\mathrm{H}_{2} \mathrm{O}(10.0 \mathrm{ml})$. The mixture was heated at $363 \mathrm{~K}$ for $3 \mathrm{~d}$. On cooling light-yellow crystals had formed.

\section{S2. Refinement}

The $\mathrm{H}$ atoms of the benzene rings were placed in idealized positions and constrained to ride on their parent atoms, with $\mathrm{C}$ $-\mathrm{H}=0.93 \AA$ and $U_{\text {iso }}(\mathrm{H})=1.2 U_{\mathrm{eq}}(\mathrm{C})$. The hydroxyl $\mathrm{H}$ atoms of the carboxyl groups were placed in ideal positions with the $\mathrm{O}-\mathrm{H}$ bond trans to the longest bond of the adjacent atom $(\mathrm{O}-\mathrm{H}=0.82 \AA)$ and refined using a riding model. One $\mathrm{H}$ atom of the water molecule were located in difference map, the other placed in an ideal position in order that reasonable hydrogen bonding is found. Finally they were refined using a riding model with $\mathrm{O}-\mathrm{H}=0.85 \AA$. All $\mathrm{O}-\mathrm{H} \mathrm{H}$ atoms were refined with $U_{\text {iso }}(\mathrm{H})=1.2 U_{\text {eq }}(\mathrm{O})$. 


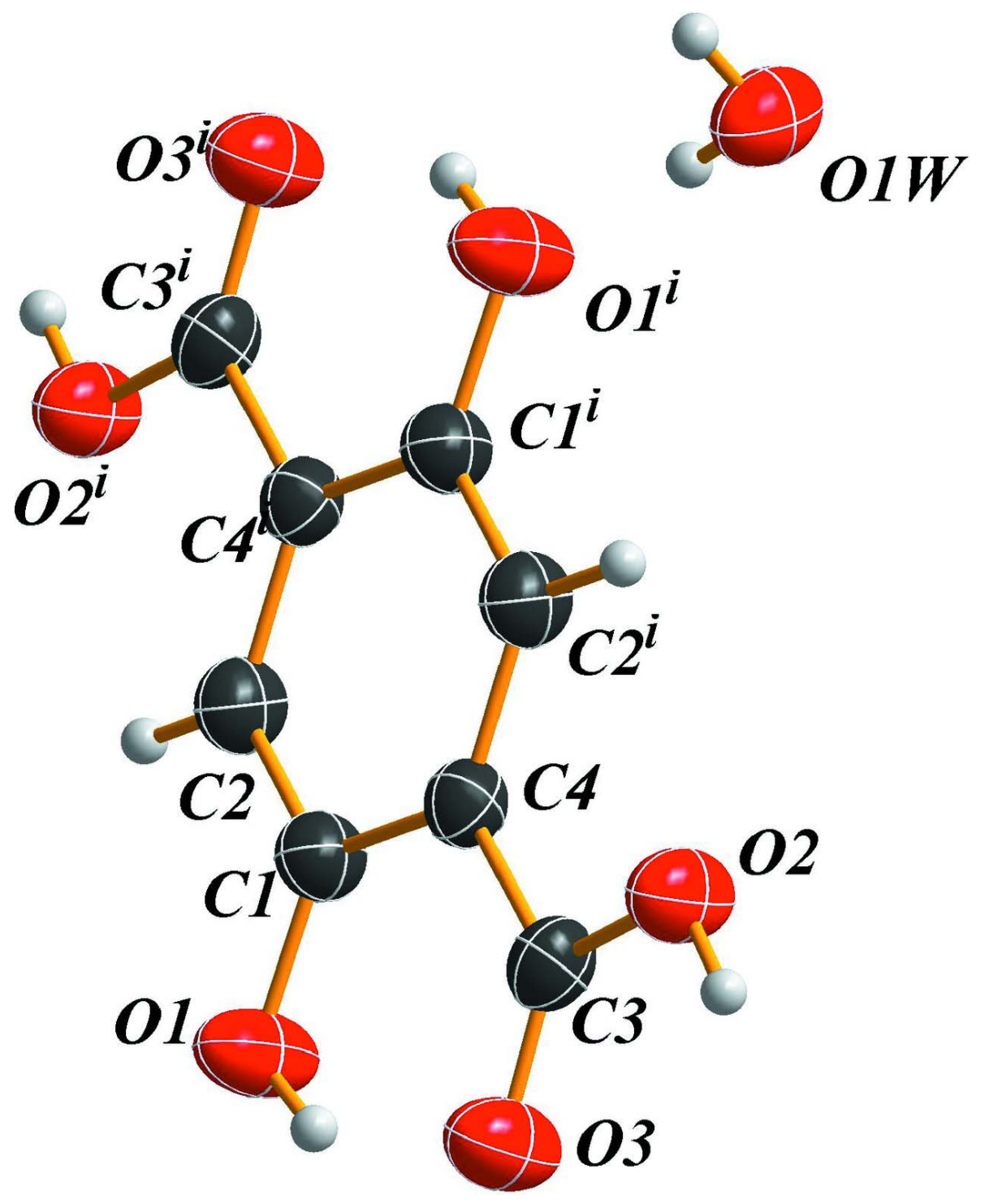

Figure 1

The molecular structure view of the title compound with labelling and displacement ellipsoids drawn at the $50 \%$ probability level. [symmetry codes: (i) $-x, 1-y, 2-z$ ]. 


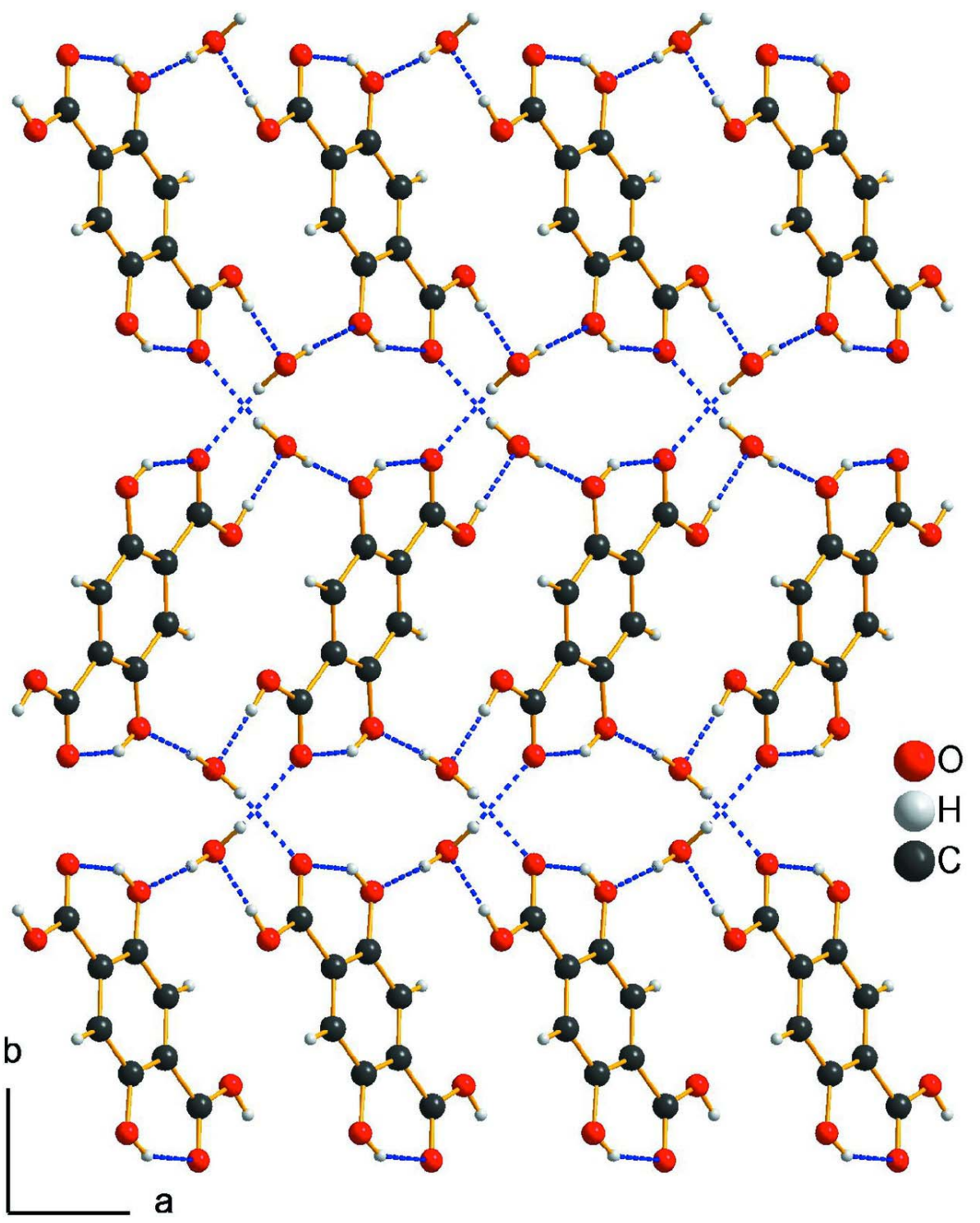

Figure 2

Crystal structure of title compound with view along $a$-axis. Hydrogen bonding is shown as blue dashed lines.

2,5-dihydroxybenzene-1,4-dicarboxylic acid dihydrate

\section{Crystal data}

$\mathrm{C}_{8} \mathrm{H}_{6} \mathrm{O}_{6} \cdot 2 \mathrm{H}_{2} \mathrm{O}$

$M_{r}=234.16$

Monoclinic, $P 2{ }_{1} / c$

$a=5.1883(10) \AA$

$b=17.545(4) \AA$

$c=5.4990(12) \AA$

$\beta=103.03(1)^{\circ}$

$V=487.68(17) \AA^{3}$

$Z=2$
$F(000)=244$

$D_{\mathrm{x}}=1.595 \mathrm{Mg} \mathrm{m}^{-3}$

Mo $K \alpha$ radiation, $\lambda=0.71073 \AA$

Cell parameters from 760 reflections

$\theta=2.3-22.5^{\circ}$

$\mu=0.15 \mathrm{~mm}^{-1}$

$T=295 \mathrm{~K}$

Tablular, light-yellow

$0.25 \times 0.20 \times 0.20 \mathrm{~mm}$ 


\section{Data collection}

Bruker APEXII CCD

diffractometer

Radiation source: fine-focus sealed tube

Graphite monochromator

Detector resolution: 8.3333 pixels $\mathrm{mm}^{-1}$

$\varphi$ and $\omega$ scans

Absorption correction: multi-scan

(SADABS; Bruker, 2009)

$T_{\min }=0.945, T_{\max }=0.963$

Refinement

Refinement on $F^{2}$

Least-squares matrix: full

$R\left[F^{2}>2 \sigma\left(F^{2}\right)\right]=0.061$

$w R\left(F^{2}\right)=0.193$

$S=1.02$

1208 reflections

73 parameters

0 restraints

Primary atom site location: structure-invariant direct methods
4475 measured reflections

1208 independent reflections

589 reflections with $I>2 \sigma(I)$

$R_{\text {int }}=0.080$

$\theta_{\max }=28.4^{\circ}, \theta_{\min }=2.3^{\circ}$

$h=-5 \rightarrow 6$

$k=-23 \rightarrow 23$

$l=-7 \rightarrow 4$

Secondary atom site location: difference Fourier map

Hydrogen site location: inferred from

neighbouring sites

$\mathrm{H}$-atom parameters constrained

$w=1 /\left[\sigma^{2}\left(F_{\mathrm{o}}^{2}\right)+(0.0851 P)^{2}\right]$

where $P=\left(F_{\mathrm{o}}{ }^{2}+2 F_{\mathrm{c}}{ }^{2}\right) / 3$

$(\Delta / \sigma)_{\max }=0.009$

$\Delta \rho_{\max }=0.36 \mathrm{e} \AA^{-3}$

$\Delta \rho_{\min }=-0.32$ e $\AA^{-3}$

\section{Special details}

Geometry. All e.s.d.'s (except the e.s.d. in the dihedral angle between two 1.s. planes) are estimated using the full covariance matrix. The cell e.s.d.'s are taken into account individually in the estimation of e.s.d.'s in distances, angles and torsion angles; correlations between e.s.d.'s in cell parameters are only used when they are defined by crystal symmetry. An approximate (isotropic) treatment of cell e.s.d.'s is used for estimating e.s.d.'s involving 1.s. planes.

Refinement. Refinement of $F^{2}$ against ALL reflections. The weighted $R$-factor $w R$ and goodness of fit $S$ are based on $F^{2}$, conventional $R$-factors $R$ are based on $F$, with $F$ set to zero for negative $F^{2}$. The threshold expression of $F^{2}>\sigma\left(F^{2}\right)$ is used only for calculating $R$-factors (gt) etc. and is not relevant to the choice of reflections for refinement. $R$-factors based on $F^{2}$ are statistically about twice as large as those based on $F$, and $R$ - factors based on ALL data will be even larger.

Fractional atomic coordinates and isotropic or equivalent isotropic displacement parameters $\left(\AA^{2}\right)$

\begin{tabular}{lllll}
\hline & $x$ & $y$ & $z$ & $U_{\text {iso }}^{*} / U_{\text {eq }}$ \\
\hline O1 & $0.0253(5)$ & $0.35101(13)$ & $0.8449(4)$ & $0.0561(7)$ \\
H1A & -0.0615 & 0.3231 & 0.9153 & $0.084^{*}$ \\
O2 & $0.4188(5)$ & $0.59034(13)$ & $0.6391(4)$ & $0.0488(7)$ \\
H2B & 0.4915 & 0.6261 & 0.5857 & $0.073^{*}$ \\
O3 & $0.2796(5)$ & $0.68281(14)$ & $0.8554(4)$ & $0.0536(7)$ \\
O1W & $0.6537(4)$ & $0.69917(13)$ & $1.4663(4)$ & $0.0524(7)$ \\
H1WA & 0.5399 & 0.7288 & 1.3796 & $0.079^{*}$ \\
H1WB & 0.7486 & 0.6811 & 1.3724 & $0.079^{*}$ \\
C1 & $0.0087(6)$ & $0.42428(18)$ & $0.9234(5)$ & $0.0371(8)$ \\
C2 & $0.1432(6)$ & $0.48027(18)$ & $0.8263(5)$ & $0.0394(9)$ \\
H2A & 0.2394 & 0.4671 & 0.7089 & $0.047^{*}$ \\
C3 & $0.2843(6)$ & $0.61572(19)$ & $0.7961(5)$ & $0.0378(8)$ \\
C4 & $0.1382(6)$ & $0.55608(17)$ & $0.9001(5)$ & $0.0337(8)$ \\
& & & & \\
\hline
\end{tabular}


Atomic displacement parameters $\left(\AA^{2}\right)$

\begin{tabular}{lllllll}
\hline & $U^{11}$ & $U^{22}$ & $U^{33}$ & $U^{12}$ & $U^{13}$ & $U^{23}$ \\
\hline O1 & $0.0761(17)$ & $0.0361(15)$ & $0.0720(15)$ & $-0.0063(12)$ & $0.0504(14)$ & $-0.0070(12)$ \\
O2 & $0.0610(15)$ & $0.0415(15)$ & $0.0545(14)$ & $-0.0033(11)$ & $0.0352(12)$ & $0.0004(11)$ \\
O3 & $0.0678(17)$ & $0.0402(15)$ & $0.0639(16)$ & $-0.0083(12)$ & $0.0380(13)$ & $-0.0066(12)$ \\
O1W & $0.0646(16)$ & $0.0461(16)$ & $0.0575(14)$ & $0.0068(12)$ & $0.0371(13)$ & $0.0091(12)$ \\
C1 & $0.0391(18)$ & $0.037(2)$ & $0.0386(16)$ & $0.0007(14)$ & $0.0155(14)$ & $-0.0015(14)$ \\
C2 & $0.0412(19)$ & $0.043(2)$ & $0.0399(17)$ & $0.0012(15)$ & $0.0208(15)$ & $-0.0001(15)$ \\
C3 & $0.0377(18)$ & $0.041(2)$ & $0.0366(17)$ & $-0.0011(15)$ & $0.0131(14)$ & $0.0051(15)$ \\
C4 & $0.0338(17)$ & $0.0363(19)$ & $0.0324(15)$ & $0.0026(13)$ & $0.0105(13)$ & $0.0015(13)$ \\
\hline
\end{tabular}

Geometric parameters $\left(\AA,{ }^{\circ}\right)$

\begin{tabular}{llll}
\hline $\mathrm{O} 1-\mathrm{C} 1$ & $1.365(4)$ & $\mathrm{C} 1-\mathrm{C} 2$ & $1.380(4)$ \\
$\mathrm{O} 1-\mathrm{H} 1 \mathrm{~A}$ & 0.8200 & $\mathrm{C} 1-\mathrm{C} 4$ & $1.405(4)$ \\
$\mathrm{O} 2-\mathrm{C} 3$ & $1.305(3)$ & $\mathrm{C} 2-\mathrm{C} 4$ & $1.393(4)$ \\
$\mathrm{O} 2-\mathrm{H} 2 \mathrm{~B}$ & 0.8200 & $\mathrm{C} 2-\mathrm{H} 2 \mathrm{~A}$ & 0.9300 \\
$\mathrm{O} 3-\mathrm{C} 3$ & $1.223(4)$ & $\mathrm{C} 3-\mathrm{C} 4$ & $1.480(4)$ \\
$\mathrm{O} 1 \mathrm{~W}-\mathrm{H} 1 \mathrm{WA}$ & 0.8485 & $\mathrm{C} 4-\mathrm{C} 1^{\mathrm{i}}$ & $1.406(4)$ \\
$\mathrm{O} 1 \mathrm{~W}-\mathrm{H} 1 \mathrm{WB}$ & 0.8511 & & \\
& & & 119.2 \\
$\mathrm{C} 1-\mathrm{O} 1-\mathrm{H} 1 \mathrm{~A}$ & 109.5 & $\mathrm{C} 4-\mathrm{C} 2-\mathrm{H} 2 \mathrm{~A}$ & $123.4(3)$ \\
$\mathrm{C} 3-\mathrm{O} 2-\mathrm{H} 2 \mathrm{~B}$ & 109.5 & $\mathrm{O} 3-\mathrm{C} 3-\mathrm{O} 2$ & $122.3(3)$ \\
$\mathrm{H} 1 \mathrm{WA}-\mathrm{O} 1 \mathrm{~W}-\mathrm{H} 1 \mathrm{WB}$ & 108.2 & $\mathrm{O} 3-\mathrm{C} 3-\mathrm{C} 4$ & $114.3(3)$ \\
$\mathrm{O} 1-\mathrm{C} 1-\mathrm{C} 2$ & $118.3(3)$ & $\mathrm{O} 2-\mathrm{C} 3-\mathrm{C} 4$ & $12.0(3)$ \\
$\mathrm{O} 1-\mathrm{C} 1-\mathrm{C} 4$ & $\mathrm{C} 2-\mathrm{C} 4-\mathrm{C} 1^{\mathrm{i}}$ & $119.9(3)$ \\
$\mathrm{C} 2-\mathrm{C} 1-\mathrm{C} 4$ & $122.1(3)$ & $\mathrm{C} 2-\mathrm{C} 4-\mathrm{C} 3$ & \\
$\mathrm{C} 1-\mathrm{C} 2-\mathrm{C} 4$ & $119.5(3)$ & $\mathrm{C} 1-\mathrm{C} 4-\mathrm{C} 3$ & \\
$\mathrm{C} 1-\mathrm{C} 2-\mathrm{H} 2 \mathrm{~A}$ & $121.5(3)$ & &
\end{tabular}

Symmetry code: (i) $-x,-y+1,-z+2$.

Hydrogen-bond geometry $\left(A,{ }^{\circ}\right)$

\begin{tabular}{lllll}
\hline$D-\mathrm{H} \cdots A$ & $D-\mathrm{H}$ & $\mathrm{H} \cdots A$ & $D \cdots A$ & $D-\mathrm{H} \cdots A$ \\
\hline $\mathrm{O} 1-\mathrm{H} 1 A \cdots \mathrm{O} 3^{\mathrm{i}}$ & 0.82 & 1.88 & $2.597(3)$ & 146 \\
$\mathrm{O} 2-\mathrm{H} 2 B \cdots \mathrm{O} 1 W^{\mathrm{ii}}$ & 0.82 & 1.74 & $2.561(3)$ & 177 \\
$\mathrm{O} 1 W-\mathrm{H} 1 W B \cdots \mathrm{O} 1^{\mathrm{iii}}$ & 0.85 & 1.94 & $2.786(3)$ & 175.0 \\
$\mathrm{O} 1 W-\mathrm{H} 1 W A^{\mathrm{ii}}{ }^{\text {iv }}$ & 0.85 & 2.04 & $2.809(3)$ & 150.4 \\
\hline
\end{tabular}

Symmetry codes: (i) $-x,-y+1,-z+2$; (ii) $x, y, z-1$; (iii) $-x+1,-y+1,-z+2$; (iv) $x,-y+3 / 2, z+1 / 2$. 